\title{
Latitudinal gradient in the taxonomic composition of parasite communities
}

\author{
R. Poulin ${ }^{1 *}$ and T.L.F Leung ${ }^{2}$ \\ ${ }^{1}$ Department of Zoology, University of Otago, PO Box 56, Dunedin 9054, \\ New Zealand: ${ }^{2}$ Zoology, School of Environmental and Rural Science, \\ University of New England, Armidale, NSW 2351, Australia
}

(Accepted 5 September 2010; First Published Online 12 November 2010)

\begin{abstract}
Although latitudinal gradients in diversity have been well studied, latitudinal variation in the taxonomic composition of communities has received less attention. Here, we use a large dataset including 950 surveys of helminth endoparasite communities in 650 species of vertebrate hosts to test for latitudinal changes in the relative contributions of trematodes, cestodes, nematodes and acanthocephalans to parasite assemblages. Although the species richness of helminth communities showed no consistent latitudinal variation, their taxonomic composition varied as a function of both host type and latitude. First, trematodes and acanthocephalans accounted for a higher proportion of species in helminth communities of fish, whereas nematodes achieved a higher proportion of the species in communities of bird and especially mammal hosts. Second, the proportion of trematodes in helminth communities of birds and mammals increased toward higher latitudes. Finally, the proportion of nematodes per community increased toward lower latitudes regardless of the type of host. We present tentative explanations for these patterns, and argue that new insights in parasite community ecology can be gained by searching for latitudinal gradients not only in parasite species richness, but also in the taxonomic composition of parasite assemblages.
\end{abstract}

\section{Introduction}

Latitudinal gradients in species diversity are among the best-studied biogeographical patterns (Willig et al., 2003). Several studies to date have investigated the relationship between latitude and parasite species diversity (Rohde \& Heap, 1998; Choudhury \& Dick, 2000; Poulin, 2001; Poulin \& Morand, 2004). However, there have been no serious attempts to document how the composition, not the richness, of parasite communities changes as a function of latitude. All else being equal, the types of parasites making up the helminth community of a vertebrate host in temperate areas may differ from those in a similar vertebrate in the tropics, because many environmental variables correlate with latitude, including the diversity and availability of intermediate hosts.

*E-mail: robert.poulin@stonebow.otago.ac.nz
As an example, we can consider how latitude might correlate with the contribution of trematodes to the parasite communities of vertebrates. Trematodes require a mollusc, usually a gastropod, as first intermediate host, and often another invertebrate as second intermediate host before they reach a vertebrate host. Generally speaking, the diversity of molluscs increases toward the equator (Macpherson, 2002; Witman \& Roy, 2009). Given the strict host specificity of trematodes for their mollusc host, we might expect that the greater the number of gastropods in an area, the higher the total number of trematode species present. Thus, all else being equal, the proportion of trematodes in the parasite communities of tropical vertebrates could be higher than in similar hosts living at higher latitudes. The validity of such predictions, however, relies on several assumptions, and empirical data are necessary to determine whether the proportion of any taxonomic group of parasites changes as a function of latitude. 
Other factors can also influence the composition of parasite communities. For instance, species-rich communities may tend to be dominated by small-bodied parasite taxa, since space saturation might constrain the body size of new species that could colonize an existing community (Poulin et al., 2008). For instance, in a relatively speciesrich helminth community, further diversification may only be possible via the addition of small parasites like trematodes and not large ones like cestodes. In addition, the nature of the host can affect the composition of its parasite community. Hosts and parasites often share a long co-evolutionary history, such that the parasite taxa found in an extant host are, to a large degree, inherited from the host's ancestor (Brooks \& McLennan, 1993). This legacy, combined with the host's ecological traits that determine what kinds of parasites it is exposed to, shape its parasite fauna. Furthermore, marked diversification of particular parasites is often only achieved in specific types of hosts. For example, some nematodes have radiated extensively into 'species flocks' in tortoises and kangaroos (Schad, 1963; Beveridge \& Spratt, 1996; Beveridge et al., 2002), but this sort of diversification has not been reported in fish or bird hosts. Therefore, in addition to latitude, the type of host used and the species richness of the community may also influence the relative contribution of major taxa to species composition of parasite communities.

Here, we investigate the determinants of taxonomic composition of parasite communities in vertebrate hosts. We limit our analyses to adult and larval stages of helminth endoparasites (trematodes, cestodes, nematodes and acanthocephalans) of fish, birds and mammals, since these have been the subjects of numerous investigations. We address the following questions: (1) Does the taxonomic composition of helminth communities show any latitudinal gradients? (2) Is taxonomic composition different in species-rich helminth communities than in those with fewer species? (3) What are the quantitative differences in taxonomic composition between the helminth communities of fish, birds and mammals? Our analyses take advantage of a very large dataset to extract general patterns that may shed light on the factors governing the assembly of parasite communities over evolutionary time scales.

\section{Material and methods}

We used a dataset compiled by Poulin \& Leung (2010) from published surveys of endohelminth communities in vertebrates. This compilation provides a large, representative and unbiased sample derived from 545 studies published between 1936 and 2009. The dataset only includes studies that provided a clear list of all species of trematodes, cestodes, nematodes and acanthocephalans found in one sample of individual hosts from the same species. Therefore, each entry in the dataset corresponds to one parasite survey for one host species from one study, i.e. one local parasite community; some host species have been the subject of a few separate studies, and thus for those host species there are two or more entries in the dataset. For each entry, the following information was recorded: (1) the name of the host species; (2) the host type, i.e. fish, bird or mammal, and the taxonomic order to which the host species belonged (based on information at www.fishbase.org); (3) the total number of endohelminth species, as well as the numbers of trematode, cestode, nematode and acanthocephalan species; and (4) the latitude at which the host sample was collected, or the latitude at the centre of the area where the hosts were obtained if they came from a region rather than a precise locality. Most available surveys came from the northern hemisphere. Thus, in preliminary analyses latitude was treated in three different ways: by using latitude regardless of whether it was north or south, by assigning negative values to southern latitudes to differentiate them from northern ones, and by using only northern latitudes. Since the effect of latitude emerging from the analyses was the same whichever of these three approaches was used, below we only present results from analyses that used latitude regardless of whether it was north or south.

Studies of parasite species richness generally correct for sampling effort, since the more hosts are examined in a particular survey, the more parasite species are found (Walther et al., 1995). However, here sampling effort was not treated as a confounding variable since the analyses did not focus on how many species were found, but on what taxon the species that were found belonged to; preliminary analyses showed that sampling effort did not affect the proportions of species belonging to different groups (correlations with proportions of species belonging to each of the four groups: all $P>0.23$ ).

The proportion of parasite species in a community that belong to higher groups like trematodes or nematodes is, in part, a biological property of the host species, because of host-parasite co-evolutionary history, and, in part, a property of the environment, which determines the presence of essential intermediate hosts and the survivorship of infective stages. Host phylogeny within fish, birds and mammals may thus influence the composition of parasite communities. Therefore, in the absence of a comprehensive species-level phylogeny of vertebrates, we included host taxonomic order as a variable in the analyses to account for possible higher-order phylogenetic influences. Furthermore, some host species contribute two or more entries in the dataset, and to avoid potential pseudoreplication, the analysis was conducted both at the survey level (across all entries in the dataset) and at the host species level. For the latter analysis, for each host species with two or more entries in the dataset, only the survey with the highest number of parasite species was retained as the most representative of the parasite fauna of that host species; other entries for that host were excluded from the hostspecies-level analyses.

There were therefore two levels of analysis (across all surveys, and across host species) for each of four measures of taxonomic composition (proportion of trematodes, of cestodes, of nematodes and of acanthocephalans per community), for a total of eight versions of the analysis of the determinants of the taxonomic composition of parasite communities. For each of these eight analyses, we used generalized linear models (GLM) implemented with the statistical software JMP 7.0 (SAS Institute Inc., Cary, North Carolina, USA). Since the 


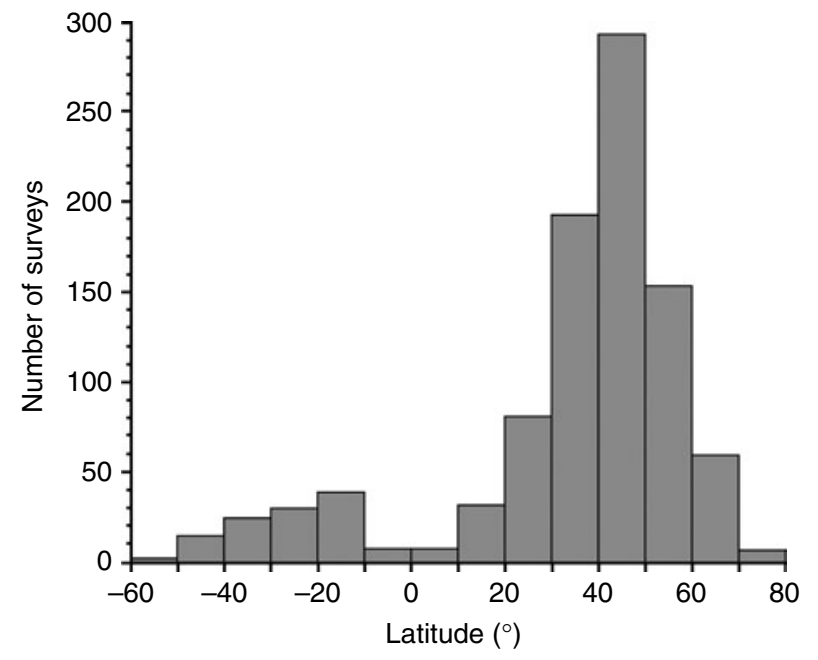

Fig. 1. Frequency distribution of latitudes at which helminth communities were surveyed, across 950 surveys in fish, bird and mammal hosts. Negative values represent southern latitudes, and positive values represent northern ones.

response variable was always a proportion, i.e. the proportion of species belonging to a particular parasite group, all models had a binomial error structure and logit link function. The explanatory variables included in the starting models were: host type (fish, bird or mammal), host order (nested within host type), parasite species richness (or the $\log _{10}$-transformed total number of helminth taxa in the community), latitude, and the interaction between host type and latitude. After starting with a full model, significance levels were based on the deviance explained by each factor, based on $\chi^{2}$ statistics, following backward stepwise elimination of unimportant $(P>0.10)$ terms. Only the final models are shown in the results.

\section{Results}

The dataset included a total of 950 helminth endoparasite communities from 650 different host species (see Poulin \& Leung, 2010, for the full dataset). The surveys consisted of 419 on fish hosts, 161 on bird hosts and 370 on mammal hosts. The parasite species richness in these surveys ranged from 1 to 52 helminth species per community, with a median of eight species (0-28 trematodes, $0-45$ cestodes, $0-40$ nematodes and 0-10 acanthocephalans). The surveys were conducted between $78^{\circ} \mathrm{N}$ and $80^{\circ} \mathrm{S}$, though the vast majority (828 out of 950) were from the northern hemisphere, and most were from temperate latitudes (fig. 1). We found no overall latitudinal gradient in parasite species richness (latitude versus log no. helminth species per survey: $r=0.046, P=0.153)$; when the four helminth groups were treated separately, only very weak correlations were obtained (all $r<0.13$ ). In other words, the number of parasite species per community showed no consistent variation as a function of latitude, and therefore any change in taxonomic composition (see below) is independent of latitudinal gradients in richness.

In the GLMs testing for the determinants of species composition in helminth community, the effect of host taxonomic order (nested within host type) only appeared influential in analyses of the proportion of trematode species per community. The influence of host order only emerged in preliminary tests using specific subsets of factors, and it was not retained in our final model which started with all factors prior to backward elimination. Therefore, the results below are unlikely to be masked by any influence of higher-order host taxonomic affiliation on the inclusion of trematodes in helminth communities. Host taxonomic order had no detectable effect on the proportions of other types of helminths (cestodes, nematodes, acanthocephalans) among the surveys included in our analyses.

Almost identical results were obtained in the GLMs whether they were carried out across all surveys, or across host species, i.e. including a single survey per host species (table 1). Parasite species richness was not retained in any of the final models. However, both host type and latitude significantly correlated with the proportion of helminth species belonging to various groups (table 1). In general, helminth communities in fish included a higher proportion of trematodes and acanthocephalans than those in other host types, whereas communities in birds and especially mammals comprised

Table 1. Results of generalized linear models of the factors influencing taxonomic composition in helminth communities of vertebrates. Eight analyses are shown, at two levels (across all surveys, across host species) and for four measures of composition (proportion of trematodes, cestodes, nematodes and acanthocephalans). The $\chi^{2}$ tests assess the significance of the deviance explained by each factor. df, Degrees of freedom.

\begin{tabular}{|c|c|c|c|c|c|c|}
\hline \multirow[b]{2}{*}{ Taxonomic composition } & \multirow[b]{2}{*}{ Factor } & \multirow[b]{2}{*}{$\mathrm{df}$} & \multicolumn{2}{|c|}{ Across surveys } & \multicolumn{2}{|c|}{$\begin{array}{c}\text { Across } \\
\text { host species }\end{array}$} \\
\hline & & & $x^{2}$ & $P$ & $x^{2}$ & $P$ \\
\hline \multirow[t]{3}{*}{ Proportion of trematodes } & Host type & 2 & 40.04 & 0.0001 & 28.24 & 0.0001 \\
\hline & Latitude & 1 & 3.04 & 0.081 & 3.01 & 0.088 \\
\hline & Host type $\times$ latitude & 2 & 5.11 & 0.078 & 5.34 & 0.069 \\
\hline Proportion of cestodes & None & - & - & - & - & - \\
\hline \multirow[t]{2}{*}{ Proportion of nematodes } & Host type & 2 & 53.85 & 0.0001 & 39.16 & 0.0001 \\
\hline & Latitude & 1 & 10.82 & 0.0010 & 7.27 & 0.0070 \\
\hline Proportion of acanthocephalans & Host type & 2 & 32.53 & 0.0001 & 17.63 & 0.0001 \\
\hline
\end{tabular}




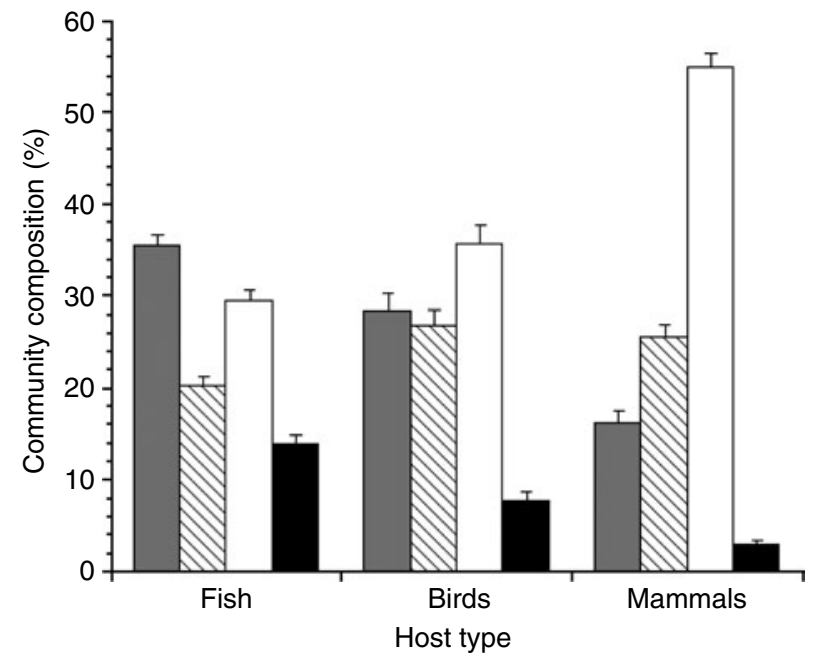

Fig. 2. Mean $( \pm \mathrm{SE})$ composition of parasite communities with respect to the proportions of trematodes (shaded bars), cestodes (hatched bars), nematodes (white bars) and acanthocephalans (black bars), in three types of vertebrate hosts, fish (419 surveys), birds (161 surveys) and mammals (370 surveys).

a higher proportion of nematodes than those in fish (fig. 2). The proportion of trematodes in helminth communities tended to be higher at higher latitudes, though this effect was not quite significant (table 1, fig. 3).
This pattern was subject to a weak interaction between host type and latitude (table 1), resulting mainly from the fact that the proportion of trematodes per community shows practically no variation in fish hosts, but tends toward its lowest values in the tropics for bird and mammal hosts. However, there was a clear and significant increase in the proportion of nematodes per community toward lower latitudes (fig. 3). Indeed, nematodes make up nearly half of the species in helminth communities in the tropics, independently of host type.

\section{Discussion}

As reported in other studies (Rohde \& Heap, 1998; Choudhury \& Dick, 2000; Poulin, 2001), the number of endohelminth parasite species per community showed no consistent variation as a function of latitude; in other words, there was no general gradient in parasite diversity among the 950 surveys included in our study. However, we found some relatively clear patterns in the taxonomic composition of helminth communities: (1) trematodes and acanthocephalans account for a higher proportion of species in helminth communities of fish, whereas nematodes represent a higher proportion of the species in communities of bird and especially mammal hosts; (2) the proportion of trematodes in helminth communities of birds and mammals increased toward higher latitudes; and (3) the proportion of nematodes per community increased toward lower latitudes regardless of the type of host.

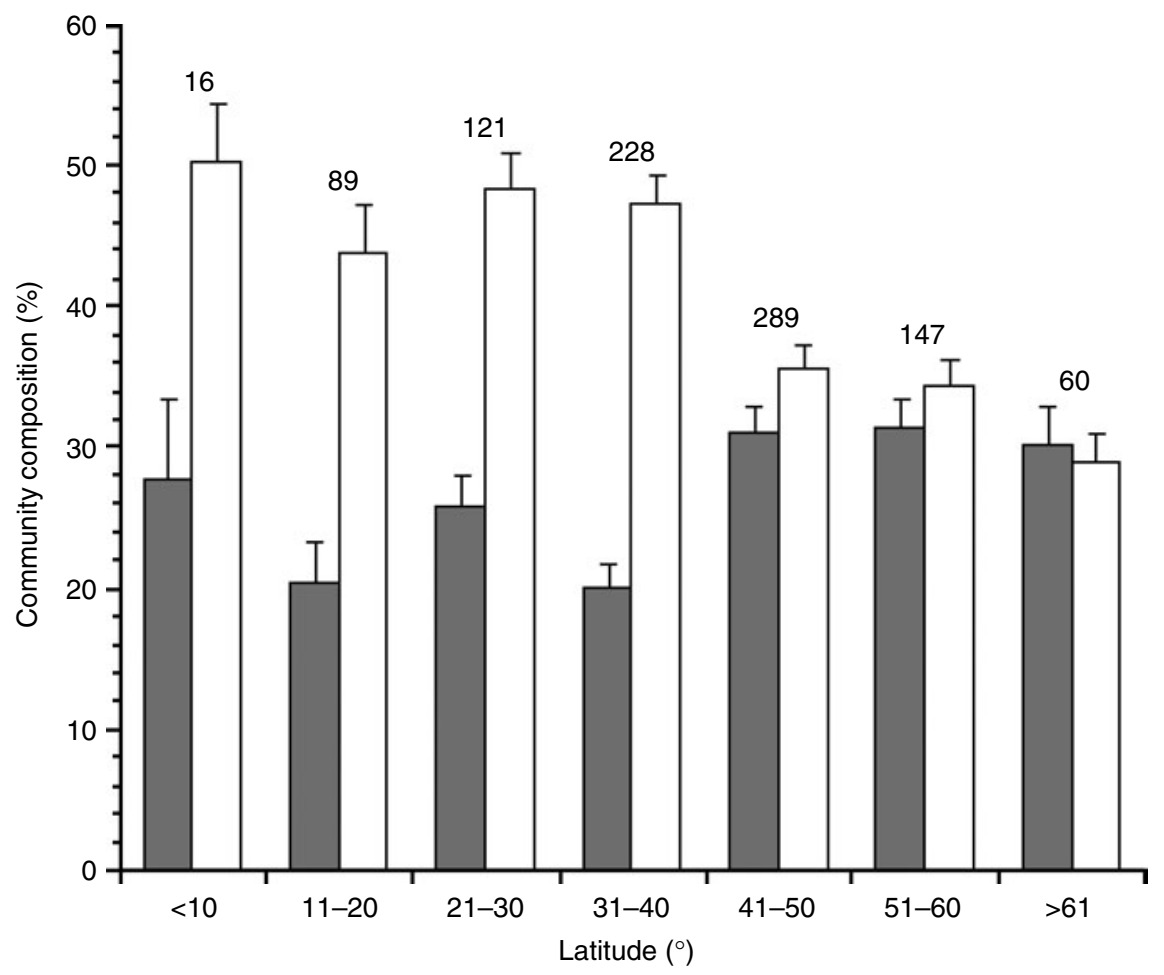

Fig. 3. Mean $( \pm \mathrm{SE})$ proportions of trematodes (shaded bars) and nematodes (white bars) in parasite communities of vertebrates, as a function of the latitude at which the community occurs. Numbers above the bars indicate the number of communities surveyed in each latitudinal band. 
Trematodes use snails and often a second intermediate host before reaching their vertebrate definitive host, whereas acanthocephalans use an arthropod, commonly a crustacean, as intermediate host. Most of these intermediate host taxa are aquatic, which may account for the fact that these two parasite groups account for higher proportions of helminth communities in fish hosts than in birds and especially mammals. In contrast, nematodes use a variety of life cycles, many of which require no intermediate hosts. Anderson (1996) noted that fish have generally few nematode parasites compared to terrestrial vertebrates. He attributed this to the terrestrial origins of parasitic nematodes, and their relatively slow colonization of aquatic habitats. Our large-scale quantitative evidence supports Anderson's (1996) qualitative observation. Nematodes are particularly dominant in communities of mammalian hosts. It is worth noting that radiations and species flocks of parasitic nematodes have only been reported from large grazing mammals, such as elephants, horses and kangaroos (Inglis, 1971; Bucknell et al., 1996; Beveridge \& Spratt, 1996; Beveridge et al., 2002), and never from birds or fish. Such radiations can result in nematodes accounting for a substantial proportion of the parasites in a community.

Because the diversity of molluscs generally increases toward the equator (Macpherson, 2002; Witman \& Roy, 2009), one obvious prediction was that they may harbour a richer fauna of trematodes, which could translate into a high proportion of trematodes in the helminth communities of definitive hosts. In fact, we found the opposite: trematodes account for a slightly higher proportion of species in helminth communities at higher latitudes, and not in the tropics. Interestingly, a study of trematode parasitism in their snail intermediate hosts has found that snails from high latitude areas tend to harbour slightly richer trematode communities than those of low latitude areas (Poulin \& Mouritsen, 2003). In contrast, small crustaceans serving as second intermediate hosts for trematodes harbour richer trematode communities at low latitudes than in high latitude areas (Thieltges et al., 2009). These contrasting latitudinal patterns in different hosts, i.e. for different stages in the life cycles of trematodes, are difficult to reconcile. Although trematode species richness in crustacean intermediate hosts is higher at low than at high latitudes, perhaps a disproportionate number of trematode species use other types of second intermediate hosts at high latitudes. It has been hypothesized that truncated life cycles, in which the second intermediate host is bypassed, are more common at high latitudes (Galaktionov \& Skirnisson, 2007); this may explain the congruence between the present results and those of Poulin \& Mouritsen (2003) and the mismatch between the present results and those of Thieltges et al. (2009). Latitudinal variation in host specificity may provide an alternative explanation. For certain types of parasites, host specificity is relaxed at higher latitudes (see Krasnov et al., 2008), and lower host specificity for the definitive hosts at high latitudes could certainly account for our results, independently of latitudinal gradients in species richness found in intermediate hosts.

We can only speculate on possible explanations for the third pattern identified by our analyses, i.e. the finding that the proportion of nematodes per community increased toward lower latitudes regardless of the type of host. One possibility is that there may be a more diverse fauna of intermediate hosts for nematodes in the tropics, and also more complex food webs, leading to a greater potential for the diversification of nematodes and their trophic acquisition by many hosts. Alternatively, warmer conditions, or less variable temperatures, at lower latitudes may favour the survival of the free-living stages of nematodes (many nematodes do not use intermediate hosts), which could promote the maintenance of a rich nematode fauna in tropical and subtropical areas, and their overrepresentation in helminth communities of vertebrates in those areas. These and other possible explanations will require further investigation. Although several questions remain unanswered, our study has highlighted the kind of novel insights that can be gained by searching for latitudinal gradients not only in parasite species richness, which appear inconsistent (Rohde \& Heap, 1998; Choudhury \& Dick, 2000; Poulin, 2001; Poulin \& Morand, 2004), but also in the taxonomic compositions of parasite assemblages.

\section{Acknowledgements}

We are grateful to Isabel Blasco-Costa and Haseeb Randhawa for useful comments on an earlier draft of this manuscript. Financial support came in part via a grant from the Marsden Fund.

\section{References}

Anderson, R.C. (1996) Why do fish have so few roundworm (nematode) parasites? Environmental Biology of Fishes 46, 1-5.

Beveridge, I. \& Spratt, D.M. (1996) The helminth fauna of Australasian marsupials: origins and evolutionary biology. Advances in Parasitology 37, 135-254.

Beveridge, I., Chilton, N.B. \& Spratt, D.M. (2002) The occurrence of species flocks in the nematode genus Cloacina (Strongyloidea: Cloacininae), parasitic in the stomachs of kangaroos and wallabies. Australian Journal of Zoology 50, 597-620.

Brooks, D.R. \& McLennan, D.A. (1993) Parascript: parasites and the language of evolution. Washington, Smithsonian Institution Press.

Bucknell, D., Hoste, H., Gasser, R.B. \& Beveridge, I. (1996) The structure of the community of strongyloid nematodes of domestic equids. Journal of Helminthology 70, 185-192.

Choudhury, A. \& Dick, T.A. (2000) Richness and diversity of helminth communities in tropical freshwater fishes: empirical evidence. Journal of Biogeography 27, 935-956.

Galaktionov, K.V. \& Skirnisson, K. (2007) New data on Microphallus breviatus Deblock \& Maillard, 1975 (Microphallidae: Digenea) with emphasis on the evolution of dixenous life cycles of microphallids. Parasitology Research 100, 963-971.

Inglis, W.G. (1971) Speciation in parasitic nematodes. Advances in Parasitology 9, 201-223.

Krasnov, B.R., Shenbrot, G.I., Khokhlova, I.S., Mouillot, D. \& Poulin, R. (2008) Latitudinal gradients in niche 
breadth: empirical evidence from haematophagous ectoparasites. Journal of Biogeography 35, 592-601.

Macpherson, E. (2002) Large-scale species richness gradients in the Atlantic Ocean. Proceedings of the Royal Society of London B 269, 1715-1720.

Poulin, R. (2001) Another look at the richness of helminth communities in tropical freshwater fish. Journal of Biogeography 28, 737-743.

Poulin, R. \& Leung, T.L.F. (2010) Taxonomic resolution in parasite community studies: are things getting worse? Parasitology 137, 1967-1973.

Poulin, R. \& Morand, S. (2004) Parasite biodiversity. Washington DC, Smithsonian Institution Press.

Poulin, R. \& Mouritsen, K.N. (2003) Large-scale determinants of trematode infections in intertidal gastropods. Marine Ecology Progress Series 254, 187-198.

Poulin, R., Beveridge, I. \& Spratt, D.M. (2008) Spatial scaling laws do not structure strongyloid nematode communities in macropodid hosts. International Journal for Parasitology 38, 1171-1177.
Rohde, K. \& Heap, M. (1998) Latitudinal differences in species and community richness and in community structure of metazoan endo- and ectoparasites of marine teleost fish. International Journal for Parasitology 28, 461-474.

Schad, G.A. (1963) Niche diversification in a parasite species flock. Nature 198, 404-406.

Thieltges, D.W., Fredensborg, B.L., Studer, A. \& Poulin, R. (2009) Large-scale patterns in trematode richness and infection levels in marine crustacean hosts. Marine Ecology Progress Series 389, 139-147.

Walther, B.A., Cotgreave, P., Price, R.D., Gregory, R.D. \& Clayton, D.H. (1995) Sampling effort and parasite species richness. Parasitology Today 11, 306-310.

Willig, M.R., Kaufman, D.M. \& Stevens, R.D. (2003) Latitudinal gradients of biodiversity: pattern, process, scale and synthesis. Annual Review of Ecology, Evolution, and Systematics 34, 273-309.

Witman, J.D. \& Roy, K. (2009) Marine macroecology. Chicago, University of Chicago Press. 